\title{
Progress in Image Management and Communication (IMAC)
}

\author{
Masayoshi Akisada, Shozo Hashimoto, Tomoho Maeda, and Eiichi Takenaka \\ IMAC 91 Organization, Kyoto, Japan \\ Seong K. Mun, Melvyn Greberman, William Hendee, and Roger Shannon \\ IMAC 89 Organization, Washington, DC
}

$\mathbf{I}^{\mathrm{M}}$ MAGE MANAGEMENT and communication in medicine has made significant progress over the past 5 years in all major fronts: clinical, technological, and administrative. Technological development has been an integral part of medicine, and new imaging modalities have revolutionized diagnostic imaging and made significant impacts on the quality of patient care. These technological innovations, however, are creating an ever increasing amount of data to be presented to physicians and to be managed over a long period of time. The technical difficulties in the distribution and management of these complex diagnostic data are universal enough that there are no national boundaries. On the other hand, health care policy and professional practice of diagnostic imaging are different in different countries. The medical imaging sector of the computer application market is small compared to the rest of the computer industry. It is desirable for the medical community to be fully informed of technological advances so that medicine can take advantage of the technologies available in the large consumer and business markets. How, then, can the diagnostic imaging community implement image management and communication (IMAC) systems using international technology to meet the needs of many different clinical environment?

The First International Conference on IMAC, which was organized in Washington, DC in June of 1989 , was attended by 500 people representing 15 countries. Experts and serious innovators from clinical, technological, and administrative disciplines participated in addressing wide ranging topics in IMAC. It also, for the first time, provided an opportunity to bring many international IMAC experts together for future collaboration. Since the first IMAC meeting, many new cooperative efforts and exchanges of ideas among the international participants have taken place. Especially for the large scale interna- tional projects in the world, a greater degree of multinational collaboration has developed. While IMAC 89 noted progress in a number of fronts, it also identified remaining challenges.

The Second IMAC conference, IMAC 91, is planned in Kyoto, Japan from April 10th through 13th, 1991. Recognizing the key challenges ahead, the second meeting will address not only international perspectives but also impressive progress in many large scale projects, as well as new innovations. More government agencies in the United States, Europe, and Japan are becoming serious about the application of IMAC technology.

The problem of diagnostic image management can be addressed in a number of ways. Availability of new technologies, such as smaller phosphor plate imaging systems and greater number of industrial participants make it possible to develop a prudent strategy for IMAC implementation.

The conference has tutorial sessions, workshops, and invited sessions as well as sessions for submitted papers and posters. A large number of experts around the world have already agreed to participate. The IMAC concept has to be compatible with radiology as well as the nonimaging practice of medicine.

There are a number of exciting and innovative IMAC related projects in Japan. The participants will be given the opportunity to arrange site visits to these projects. Site visits will be offered at the following locations: Hokkaido University Hospital, Sapporo; Keio University Hospital, Tokyo; Kochi University Hospital, Kochi; Kyoto University Hospital, Kyoto; Nagoya University Hospital, Nagoya; National Defense Medical College, Tokorozawa City; Tsukuba University Hospital, Ibaraki; and other sites to be announced.

New technical innovations, especially the potential use of high definition television from 
both industry and academia, will be discussed and exhibited.

Additional information can be obtained by contacting either Seong K. Mun, PhD, Department of Radiology, Georgetown University Hospital, 3800 Reservoir Rd, Washington, DC,
USA. Telephone: 202-687-5990, Fax 202-7843479; or Dr Eiichi Takanaka, IMAC Secretariat, No 501, Kenshu Building, Hongo 7-2-4, Bunkyo-ku, Tokyo 113, telephone: 81-33-8145451, Fax 81-33-811-0676. 